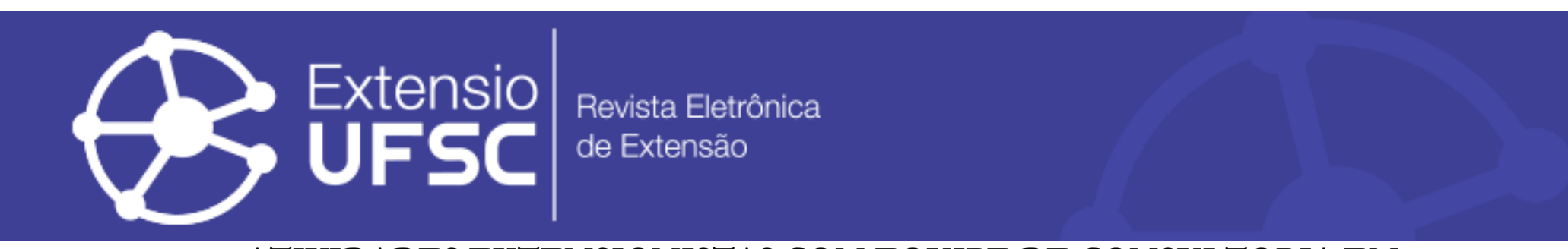

\title{
ATIVIDADES EXTENSIONISTAS COM EQUIPE DE CONSULTORIA EM CUIDADOS PALIATIVOS: CONTRIBUIÇÕES NA FORMAÇÃO EM SAÚDE
}

\author{
Franciele Roberta Cordeiro \\ Universidade Federal de Pelotas \\ franciele.cordeiro@ufpel.edu.br \\ Juliana Zeppini Giudice \\ Universidade Federal de Pelotas \\ juliana_z.g@hotmail.com \\ Carina Rabêlo Moscoso \\ Universidade Federal de Pelotas \\ carina_moscoso@hotmail.com
}

Vanessa Pellegrini Fernandes
Universidade Federal de Pelotas
nessapfernandes@gmail.com

Ana Cristina Fraga da Fonseca Universidade Federal de Pelotas anacfragafonseca@gmail.com

Julieta Carriconde Fripp

Universidade Federal de Pelotas julietafripp@gmail.com

\section{Resumo \\ Este artigo tem por objetivo relatar atividades extensionistas desenvolvidas junto a uma Equipe de Consultoria em Cuidados Paliativos (ECCP) entre 2018 e 2019. Destaca-se a organização, dinâmica de avaliação e atenção às pessoas em cuidados paliativos (CP) hospitalizadas e suas famílias pela ECCP e estudantes de graduação em enfermagem em articulação com profissionais diretamente responsáveis pela assistência em unidades de internação de um Hospital de Ensino do Sul do Brasil. Por serem enfermeiros os profissionais mais vinculados ao cuidado, seja por suas atividades ou pelo tempo que permanecem com os indivíduos, é fundamental a educação destes sobre o morrer e a morte desde a graduação. Conclui-se que ECCP em instituições hospitalares pode melhorar a qualidade do cuidado prestado às pessoas com doenças que ameaçam à vida e suas famílias, por meio da educação permanente de profissionais saúde e da aproximação de estudantes com as especificidades deste cenário. \\ Palavras-chave: Cuidados Paliativos na Terminalidade da Vida; Hospitais de Ensino; Educação em Enfermagem; Equipe de Assistência ao Paciente; Cuidados Paliativos. \\ EXTENSIONIST ACTIVITIES WITH THE PALLIATIVE CARE CONSULTING TEAM: CONTRIBUTIONS TO EDUCATION IN HEALTH}

Abstract

This article aims to report the extension activities developed with a palliative care consultation team (PCCT) between 2018 and 2019. It stands out the organization, assessment dynamics and attention to patient in palliative care (PC) and their family by PCCT and ungraduated nursing students in coordination with professionals directly responsible for assistance in the units of internment of a Teaching Hospital in southern Brazil. As nurses are the professional more linked to care, either for their activities or for the time that they spend with the patients, it is essential to educate them about dying and death since graduation. It is concluded that PCCT in hospital institutions can improve the quality of care provided to people with life threatening diseases and their families, through permanent education of health professionals and the approximation of students with the specificities of this scenario.

Keywords: Hospice Care; Hospitals Teaching Education; Nursing; Patient Care Team; Palliative Care.

\section{ACTIVIDADES EXTENSIONISTAS CON EL EQUIPO CONSULTOR DE CUIDADOS PALIATIVOS: CONTRIBUCIONES A LA FORMACIÓN EN SALUD}

\section{Resumen}

Este artículo tiene como objetivo reportar las actividades de extensión con un Equipo Consultivo de Cuidados Paliativos (ECCP) entre 2018 y 2019. Destaca la organización, dinámica de evaluación y atención a personas hospitalizadas en cuidados paliativos (CP) y sus familias por ECCP y estudiantes de licenciatura en enfermería en articulación con profesionales directamente responsables de la asistencia en las unidades de internación de un Hospital Docente del sur de Brasil. Como el enfermero es el profesional más vinculado al cuidado, ya sea por sus actividades o por el tiempo que pasa con las personas, es fundamental educarlos sobre el morir y la muerte desde la graduación. Se concluye que la ECCP en las instituciones hospitalarias puede mejorar la calidad de la atención prestada a las personas con enfermedades que amenazan la vida y sus familias, por medio de la educación permanente de los profesionales de la salud y de la aproximación de los estudiantes a las especificidades de este escenario.

Palavras clave: Cuidados Paliativos al Final de la Vida; Hospitales de Enseñanza; Educación en Enfermería. Grupo de Atención al Paciente. Cuidados Paliativos. 


\section{INTRODUÇÃO}

Os Cuidados Paliativos (CP) são uma abordagem multidisciplinar que tem por finalidade a promoção do conforto e o alívio de sintomas de pessoas com doenças graves e limitantes da vida e suas famílias (IAHPC, 2018). Essa filosofia de cuidados teve sua emergência na década de 1960, na Inglaterra, com o movimento Hospice. Nas décadas seguintes, os cuidados e direitos das pessoas em final de vida tornaram-se o centro do debate de movimentos pró-eutanásia e daqueles que reivindicavam o direito de escolha dos pacientes sobre os tratamentos e decisões em torno de seus corpos (MENEZES; BARBOSA, 2013). Os CP surgem como disciplina da área da saúde, recebendo essa denominação e tornando-se campo de conhecimento no Canadá, em 1975 (CASTRA, 2009).

No Brasil, em 2018, foi publicada a Resolução n41, que dispõe diretrizes para a organização dos cuidados paliativos no Sistema Único de Saúde (SUS) (BRASIL, 2018). Em nível estadual, Rio Grande do Sul e Goiás apresentam políticas públicas para os cuidados paliativos (RIO GRANDE DO SUL, 2019; GOIÁS, 2017).

Em relação ao local de assistência e cuidado, no Brasil, o hospital é o local para o qual as pessoas mais são encaminhadas no final da vida, devido ao aparato tecnológico e à (falta de) condições materiais, financeiras e emocionais de familiares e cuidadores (CORDEIRO; KRUSE, 2019).

No contexto hospitalar, equipes de consultoria em cuidados paliativos (ECCPs) podem oferecer suporte aos profissionais que atuam diretamente no cuidado aos pacientes com doenças que não respondem mais ao tratamento modificador e suas famílias. ECCPs são multiprofissionais e orientam as condutas a serem tomadas pela equipe que assiste e é diretamente responsável pelo paciente. Essas condutas dizem respeito às terapêuticas medicamentosas para controlar sintomas, elaboração de planos individualizados de cuidados - levando em conta as necessidades e desejos da pessoa, organização do retorno ao domicílio (quando possível), intervenções com a família, organização econômica e social para os cuidados em fim de vida e após o óbito. Estudos realizados nos Estados Unidos da América (EUA) evidenciaram que, além dos benefícios em termos de cuidados, as equipes de consultoria em cuidados paliativos reduzem despesas hospitalares, otimizando recursos, especialmente por meio da diminuição do tempo de internação (MORRISON et al., 2011; CASSEL et al., 2015).

No Brasil, ECCPs atuam majoritariamente nos hospitais, havendo escassa produção sobre tais equipes. Estudos caracterizam o perfil de pacientes acompanhados em unidades clínicas ou unidades de cuidados paliativos (BEDUSCHI et al., 2018; BASTOS et al., 2018; CASTÔR et al., 
2019). Outros relatam a implementação de serviços hospitalares, na modalidade unidade de internação (GARCIA et al., 2014; KRUSE et al., 2007; MARCUCCI et al., 2017).

Dessa forma, identifica-se necessidade de disseminação sobre ECCPs, tendo em vista que, para além do papel do consultor, tais equipes têm como missão participar da formação de estudantes e profissionais nessa área de conhecimento. Frente ao exposto, este artigo tem por objetivo relatar atividades extensionistas junto a uma ECCP entre 2018 e 2019.

\section{MATERIAIS E MÉTODOS}

Estudo descritivo, do tipo relato de experiência, com base nas ações do projeto de extensão "A consulta de enfermagem como instrumento de cuidado às pessoas com doenças que ameaçam a vida e suas famílias", em colaboração com ECCP do Hospital Escola da Universidade Federal de Pelotas/Empresa Brasileira de Serviços Hospitalares (HE UFPel/EBSERH). Os participantes das atividades foram quatro docentes, duas profissionais de saúde da ECCP, dez discentes do curso de Graduação em enfermagem e uma enfermeira discente do Programa de Pós-Graduação em Enfermagem da Universidade.

O cenário da experiência foi constituído por unidades de internação clínica e cirúrgica do HE UFPel/EBSERH. A instituição possui 175 leitos e é referência para 28 municípios da região Sul do Rio Grande do Sul. A oncologia é a área com destaque nos atendimentos da instituição, que possui ambulatório de quimioterapia, serviço de radioterapia e as unidades de internação que hospedam majoritariamente pacientes em tratamento ou com complicações oncológicas (HE UFPEL, 2018).

A experiência relatada no presente artigo consiste na realização de consultas de enfermagem direcionadas às pessoas em cuidados paliativos hospitalizadas e suas famílias. Tais consultas eram realizadas entre dois e três dias da semana - podendo ocorrer, inclusive, nos finais de semana, os quais não eram preestabelecidos e variavam de acordo com a disponibilidade dos integrantes do projeto de extensão. Em relação ao tempo de duração, cada consulta teve tempo variável e não foi possível aferir e nem realizar média do tempo dispendido a cada paciente e família. Isso porque em cada abordagem era avaliada a disponibilidade dos indivíduos. Ou seja, pacientes com maior grau de deterioração clínica, com reduzida capacidade cognitiva e menor interação, ou familiares mais sensibilizados e afetados emocional e fisicamente, tinham menor tempo de consulta. Até mesmo como forma de preservação e respeito ao tempo e conforto de cada pessoa.

As consultas eram realizadas, em sua maioria, por estudantes de graduação em enfermagem, em dupla ou individualmente, após terem realizado vivências junto à ECCP e acompanhado a 
médica e a enfermeira na abordagem ao paciente em cuidados paliativos hospitalizado e suas famílias. Em momentos pontuais, docente também realizou o acompanhamento dos estudantes, entretanto, os docentes atuaram na revisão da construção da lista de problemas, diagnósticos e prescrições de enfermagem. Também orientavam as intervenções a serem realizadas.

Ainda em relação às consultas de enfermagem, elas eram norteadas por um roteiro, baseado no processo de enfermagem e em instrumentos específicos de avaliação da funcionalidade clínica em cuidados paliativos. Tal instrumento é detalhado nos resultados. Destaca-se que por não haver, no período em que as atividades foram desenvolvidas, um sistema específico de registro das intervenções realizadas pela ECCP no prontuário dos pacientes, a lista de problemas, diagnósticos e prescrições de enfermagem eram realizadas no instrumento do projeto e armazenadas em pasta específica na sala da equipe. As prescrições eram orientadas verbalmente para implementação pela equipe ou realizadas diretamente pelos integrantes do projeto (ECCP, discentes, docente).

$\mathrm{Na}$ sala da ECCP eram mantidos os livros da taxonomia da North American Nursing Diagnosis Association - International (NANDA - I) e da Classificação das Intervenções de Enfermagem (NIC), para construção dos diagnósticos e prescrições de enfermagem, respectivamente, além de quadro com a classificação das necessidades humanas básicas, segundo proposta da teórica Wanda Horta, a qual foi eleita norteadora para o desenvolvimento do processo de enfermagem nas atividades.

Ressalta-se que as taxonomias e teórica mencionadas são utilizadas durante a formação dos estudantes de enfermagem da UFPel, acerca da sistematização da assistência de enfermagem (SAE) e também no hospital em que as atividades foram desenvolvidas.

O relato das atividades está estruturado da seguinte forma: primeiro, será apresentada a maneira como está configurada a equipe de consultoria em cuidados paliativos, bem como sua dinâmica de funcionamento, com vistas a favorecer sua identificação e inspirar outros profissionais e serviços de saúde a implementarem esse tipo de equipe de cuidados paliativos em outros hospitais brasileiros; posteriormente, será relatado o modo como ocorre a integração ensino/serviço, favorecendo a formação de estudantes da área da saúde, especialmente enfermagem e medicina, para os cuidados paliativos.

\section{RESULTADOS E ANÁLISES}

\section{Equipe de consultoria em cuidados paliativo: dinâmica e papel pedagógico}

A ECCP é um serviço do HE UFPel/EBSERH que possui espaço físico próprio, o qual constitui-se de uma sala com computador, armários, sofá e mesa de reuniões. Ela foi criada em 2016, com o objetivo de integrar e complementar a rede de CP da cidade de Pelotas. Inicialmente, 
a equipe era composta por uma enfermeira, uma médica, uma assistente social, uma psicóloga e um capelão.

Em 2019, devido às demandas institucionais, a equipe foi reduzida a duas médicas e uma enfermeira, as quais integram permanentemente a equipe, sendo que psicólogo e assistente social são solicitados apenas em casos específicos. O capelão segue atuando como voluntário. Quanto ao tipo de vínculo, destaca-se que as médicas não atuam exclusivamente na ECCP e trabalham sob regime da EBSERH, que gerencia a instituição desde 2014. Já a enfermeira possui dedicação exclusiva à equipe, além de ter vínculo do tipo regime jurídico único com a UFPel.

Como atividade principal da ECCP, destaca-se a função conselheira aos profissionais de saúde que atuam diretamente na assistência em unidades de internação do Hospital. Em média, são solicitadas 15 novas consultorias mensalmente. Esse número pode variar, para mais ou para menos, conforme o período do ano, além do período de férias das profissionais. Depois de avaliados, os pacientes passam a ser acompanhados também pela ECCP, que orientará as decisões em relação ao plano de cuidado. Destaca-se que, embora o Hospital seja referência em oncologia, essa não é a única especialidade compreendida nos atendimentos.

No que tange ao funcionamento, a atuação da ECCP inicia quando o médico assistente responsável pelo paciente preenche uma ficha de solicitação da consultoria e encaminha ao serviço. Posteriormente, médica e enfermeira realizam conjuntamente avaliação do paciente e da família, a fim de reconhecê-los, identificar demandas de cuidado, percepções acerca do adoecimento e do prognóstico. Após o contato inicial, dialoga-se com médicos e enfermeiros responsáveis pela assistência direta na unidade de internação, de modo a orientar as decisões envolvendo prescrição de medicamentos para controle de sintomas, limitação, retirada ou não de tratamentos que prolongariam o sofrimento e não atuariam na melhora ou recuperação do indivíduo, além do estabelecimento de um plano individualizado de cuidados. Esse plano pode ser aceito e implementado pela equipe assistente responsável ou não.

Em países como a França, em que ECCP estão presentes no sistema de saúde, existe resistência dos profissionais solicitarem consultorias. Dentre os motivos, destacam-se: sentimento de impotência frente ao paciente e à família, sensação de estar tendo as capacidades técnicas e competências colocadas à prova, medo de perder o controle sobre a situação (GAMBLIN et al., 2013). No Brasil, ainda não há estudos que evidenciem os motivos dessa resistência ou limitada solicitação das equipes.

Ressalta-se que todo o processo, desde a solicitação da avaliação, a avaliação propriamente dita e o plano proposto é registrado em prontuário eletrônico. Como ferramenta para nortear a prática da equipe, desde sua constituição, foi elaborada uma ficha, a qual busca coletar informações 
sociodemográficas, de identificação pessoal, sobre as crenças, espiritualidade e religiosidade, acerca da condição clínica e história de saúde pregressa. Esse documento tem como base o modelo proposto pela Academia Nacional de Cuidados Paliativos (MACIEL, 2012).

Além desse, outros instrumentos para avaliação de sintomas e determinação do grau de funcionalidade são utilizados. Esses instrumentos são: a Escala de Avaliação de Sintomas de Edmonton (ESAS), a Escala de Performance Paliativa (PPS), a Escala de Avaliação de Performance do Eastern Cooperative Oncology Group (PS-ECOG) e a Escala de Performance de Karnofsky (KPS). A ESAS está validada para uso com pacientes com câncer avançado (MANFREDINI, 2014). A PPS foi somente traduzida para o português (SALES; CARVALHO, 2009). A ECOG e a KPS não possuem validação no Brasil, mas são comumente utilizadas na prática clínica em cuidados paliativos.

A ECCP também possui papel pedagógico na Instituição. Por estar em um Hospital Escola, ela acolhe estudantes de diferentes cursos da graduação da área da Saúde, além de estudantes da pós-graduação das residências médica e multiprofissional. Isso se dá por meio da integração ensinoserviço, em estágios curriculares ou através da cooperação por projetos de ensino, pesquisa e extensão. Também, realiza capacitação junto aos profissionais que atuam diretamente na assistência.

Destaca-se que uma das limitações em relação ao trabalho da equipe de consultoria reside no fato de que apenas o médico assistente do paciente pode solicitar o acompanhamento e avaliação. Essa postura e decisão da gestão da instituição vai de encontro aos modelos de equipe de consultoria internacionalmente reconhecidos e que permitem aos demais profissionais da equipe realizarem tal solicitação.

Estudo (PAN et al., 2017) realizado em Taiwan demonstra que enfermeiras da oncohematologia possuem maior propensão a identificar pacientes com necessidades de cuidados paliativos. Recomenda-se a incorporação de programas de capacitação às equipes de enfermagem, de modo a ampliar o conhecimento e as atitudes em relação às práticas e solicitações de ECCP. Tal ação pode garantir que pacientes em final de vida e suas famílias recebam cuidados paliativos mais abrangentes e de melhor qualidade.

Outra limitação é não haver discussões com todos os profissionais que assistem aos pacientes, ou seja, técnicos de enfermagem, auxiliares, fisioterapeutas, entre outros. Especialmente nos casos com maiores implicações éticas, indicam-se reuniões de consultas multidisciplinares, as quais envolvem todos os atores envolvidos na assistência direta e na ECCP. Essa modalidade de trabalho em equipe permite o compartilhamento de opiniões e a tomada de decisões de maneira mais dinâmica, reduzindo a sobrecarga do médico (LAVAL et al., 2008). 
Por fim, verifica-se espaço a ser explorado pela ECCP no que tange à educação permanente na instituição. Uma possibilidade é o desenvolvimento de capacitações in loco, nas unidades de internação, sobre os aspectos multidimensionais que envolvem os CP.

\section{Integração ensino-serviço e formação de estudantes para os cuidados paliativos}

Dentre as possibilidades de integração ensino-serviço está a extensão universitária. As atividades extensionistas são meios de efetivar o compromisso social das Universidades para com a sociedade (FERNANDES et al., 2012; FORPROEX, 2012). A interação se dá por meio de cursos, eventos, prestação de serviços, publicações e produtos acadêmicos e atividades propriamente ditas de extensão (MICHELON et al., 2019).

Tendo em vista que o final da vida, o morrer e a morte ainda são temas incipientes na formação acadêmica dos estudantes de enfermagem, em 2018 foi elaborado e teve início o projeto de extensão "A consulta de enfermagem como instrumento de cuidado às pessoas com doenças que ameaçam à vida e suas famílias". Tal projeto tinha como objetivo sensibilizar estudantes para realizarem consultas de enfermagem junto aos pacientes em cuidados paliativos, utilizando o processo de enfermagem. Estudo de revisão demonstrou que durante a formação e a atuação profissional em enfermagem a abordagem dos cuidados paliativos ainda é frágil devido à estrutura limitada e à falta de conhecimento sobre ferramentas que possam auxiliar no acompanhamento de pacientes e familiares (SILVA; CECCHETTTO, 2019).

No projeto de extensão, inicialmente, os acadêmicos acompanhavam a ECCP, por no mínimo 20 horas, a fim de observar o processo de tomada de decisão sobre condutas terapêuticas, o modo como se abordavam questões sobre final de vida e como se avaliavam pessoas em final de vida e suas famílias, com instrumentos padronizados ou não. Posteriormente, os acadêmicos realizavam consultas de enfermagem, norteadas por um instrumento de avaliação, produzido pelos participantes do projeto. Este instrumento tinha o intuito de guiar o atendimento ao indivíduo e a sua família e era composto, inicialmente, pela anamnese e exame físico. Na sequência, continha escalas de avaliação específicas de sintomas, funcionalidade e de aspecto psicológico, a citar: Escala Visual Analógica para Avaliação da Dor, Escala de Avaliação de Sintomas de Edmonton (ESAS), Escala de Desempenho Paliativo (PPS) e o Indicador de Risco Psicológico em Oncologia. Esse último era utilizado para determinar a necessidade de solicitação de acompanhamento psicológico e foi indicado para uso por psicólogos da instituição. Na parte final do instrumento constava o espaço para a construção da lista de problemas, diagnósticos e prescrições de enfermagem, além de imagem para identificação da localização de lesões por pressão ou lesões relativas à falha da pele e úlcera terminal de Kennedy. 
Em 18 meses, 59 pessoas com doenças avançadas, em fase final de vida ou em fase ativa de morte, foram avaliadas pelos estudantes. Eram predominantemente mulheres, casadas e com baixa escolaridade. Em relação ao diagnóstico, neoplasia foi predominante, especialmente de pulmão e mama. Tal fato pode estar atrelado ao fato de o hospital em questão ser referência em oncologia. Quanto às principais queixas dos pacientes, houve predominância da dor, dispneia, inapetência e náusea. Mais de 30\% dos pacientes possuíam alguma lesão por pressão, tendo predominado as regiões sacara, calcâneos e trocanteres enquanto localização (MEDEIROS, 2020).

A partir da anamnese e exame físico, construiu-se lista com os três principais problemas de enfermagem atrelados às NHB afetadas, sobressaindo-se oxigenação, nutrição e percepção dolorosa. Os principais diagnósticos de enfermagem elencados foram: nutrição desequilibrada: menor do que as necessidades corporais (00022), dor aguda (00132), troca de gases prejudicada (00030). Este resultado converge, em partes, com os resultados de Xavier et al.(2019), que avaliaram 73 pacientes em um Centro de Alta Complexidade em Oncologia (CACON). No estudo, em relação à dimensão física se destacaram os seguintes diagnósticos: nutrição desequilibrada: menor que as necessidades corporais, perfusão periférica ineficaz, dor crônica.

No que tange às prescrições, para tentar resolver os problemas atrelados à oxigenação, os cuidados mais prescritos foram: administrar oxigênio suplementar conforme prescrito; atentar para sinais como cianose, agitação e confusão mental; manter a cabeceira elevada a $45^{\circ}$. Para a nutrição: administrar nutrição enteral, conforme apropriado; determinar o padrão nutricional do paciente e a capacidade de atender às necessidades nutricionais; e estimular o paciente a conversar sobre as preferências alimentares com o nutricionista, quando possível. Em relação à percepção dolorosa: aplicar escala da dor antes e após analgesia; fazer avaliação abrangente da dor para incluir localização, caracterização, início/duração, frequência, qualidade, intensidade da dor e fatores precipitantes; e assegurar cuidados analgésicos para a paciente.

Vale destacar que durante o trânsito dos estudantes entre as unidades foram realizadas negociações com as equipes médicas e de enfermagem para atender outras demandas de cuidado como, por exemplo, adequação do horário do banho, retirada da contenção mecânica, liberação para velório de familiar e solicitação de atendimento odontológico.

Com relação ao desfecho, o óbito foi o principal. No entanto, dez pacientes tiveram alta para o domicílio, sem acompanhamento de serviço de atenção domiciliar, e oito tiveram alta sob essa abordagem. Um paciente teve alta da consultoria em Cuidados Paliativos (MEDEIROS, 2020).

Além das consultas, de modo a sensibilizar os profissionais e estudantes do hospital, em 2018 e 2019, no dia mundial dos cuidados paliativos, comemorado mundialmente no segundo sábado do mês de outubro, acadêmicos, docentes e profissionais elaboraram folhetos educativos 
sobre os princípios dos cuidados paliativos e os serviços que oferecem esse tipo de cuidado no município. Também foram disponibilizados painéis, a fim de que o público-alvo das ações pudesse expor seus desejos antes de morrer.

As dificuldades enfrentadas pelos estudantes estiveram associadas, em sua maioria, à baixa funcionalidade clínica dos pacientes, devido aos encaminhamentos médicos tardios à ECCP (OLIVEIRA et al., 2019). Dessa forma, verifica-se a relevância em formar profissionais de saúde com capacidade de avaliação clínica, que saibam identificar o momento oportuno para iniciar o acompanhamento sob CP.

O diagnóstico de que uma doença não responde mais ao tratamento modificador, e que esta adentra o processo de morrer, depende da progressão da doença, de parâmetros clínicos e da funcionalidade, os quais podem ser avaliados por meio de instrumentos padronizados. Para avaliação da funcionalidade, por exemplo, em cuidados paliativos, a Escala de Desempenho Paliativo (Palliative Performance Status - PPS) consegue indicar em que momento a doença começa a interferir na capacidade de realizar trabalho, manter as atividades de vida diária e os níveis de ingesta e consciência (MURAD JÚNIOR, 2011).

Na oncologia, o Índice Prognóstico Paliativo (Palliative Prognostic Index - PPI) tem sido recomendado para uso na prática clínica visando estimar a sobrevida dos pacientes e auxiliar no planejamento de cuidados adequados à funcionalidade dos pacientes e da família (FARINHOLT et al., 2018). A PPI é uma ferramenta utilizada para avaliar a expectativa de vida entre 30 dias e seis meses. Considera a presença ou ausência de cinco preditores clínicos: escore obtido na PPS, ingesta oral, presença de edema, dispneia ao repouso e delirium. Sua aplicação não se restringe aos médicos e enfermeiros, mas também pode ser aplicada pelo demais profissionais que assistem o paciente (FARINHOLT et al., 2018; YAMADA et al., 2017).

Ambas as ferramentas acima mencionadas tiveram sua validade, no Brasil, avaliada no estudo de Murad Júnior (2011). $\quad$ Além delas, para uso no país, foi validada a ferramenta Necessidades Paliativas (NECPal), a qual auxilia na identificação de pacientes que necessitam precocemente do acompanhamento de equipes especializadas em cuidados paliativos. Tal ferramenta possui, além da pergunta surpresa: "Você ficaria surpreso se este paciente morresse ao longo do próximo ano?”, indicadores clínicos específicos para grupos de doença específicos entre outros indicadores clínicos gerais (SANTANA et al, 2020).

Acredita-se que, trabalhar com tais instrumentos desde a formação, é uma importante estratégia para modificar a realidade de pessoas com doenças que ameaçam à vida e só recebem cuidados paliativos diante da fase ativa de morte, também conhecida como fase agônica, ou seja, nos dias ou horas finais que antecedem o óbito. A comunicação não verbal, delirium e sonolência, 
predominaram entre as pessoas avaliadas, limitando identificação de suas reais necessidades e desejos. Assim, igualmente, mostra-se necessário o desenvolvimento, desde a graduação, de habilidades de comunicação além da fala, a fim de identificar sintomas e intervir de maneira mais efetiva - e precoce - junto aos pacientes.

\section{CONSIDERAÇÕES FINAIS}

As ações desenvolvidas pela ECCP e pelo projeto de extensão e podem modificar a forma como estudantes e profissionais de saúde ofertam cuidados às pessoas com doenças limitantes da vida e suas famílias. Essa mudança vai ao encontro do que é proposto por políticas públicas de saúde e resoluções brasileiras que visam difundir e implementar os cuidados paliativos como modalidade de cuidado a ser ofertada no SUS.

Para os estudantes e profissionais de enfermagem, especificamente, acredita-se que a incorporação do processo de enfermagem, além de instrumentos de avaliação clínica específicos, é uma forma de contribuir na qualificação da formação e atuação profissional. Acredita-se que, para além dos cuidados paliativos, incorporar tais estratégias pode corroborar com o desenvolvimento de uma prática de cuidado mais consoante com as diretrizes internacionais e os princípios científicos, os quais fomentam o desenvolvimento do pensamento crítico e o raciocínio clínico frente ao cuidado para com pacientes e suas famílias em diferentes situações e cenários.

Como limitações para o melhor andamento das ações, destacam-se a redução no número de profissionais que compõe a equipe de consultoria, a falta de financiamento que pudesse viabilizar ações de maior impacto do projeto de extensão junto aos pacientes, especialmente no que se refere à realização de seus desejos.

Contudo, acredita-se que a experiência aqui relatada pode inspirar outros serviços brasileiros a implementarem equipes de consultoria em cuidados paliativos, as quais mantenham vínculos com os cursos de graduação e pós-graduação das Universidades, de modo a potencializar a educação para os cuidados em final de vida e cuidados paliativos desde a formação inicial, com continuidade durante a atuação profissional.

\section{REFERÊNCIA}

BASTOS, B. R. et al. Perfil sociodemográfico dos pacientes em cuidados paliativos em um hospital de referência em oncologia do estado do Pará, Brasil. Revista Pan-Amazônica de Saúde, v. 9, n. 2, p. 31-36, 2018. Disponível em: http://dx.doi.org/10.5123/s2176-62232018000200004. Acesso em: 15 abr. 2020. 
BEDUSCHI, F. M. et al. Cuidados paliativos no atendimento público hospitalar: a importância do atendimento de pacientes jovens. Revista Sociedade Brasileira de Clínica Médica, v. 16, n. 2, p. 80-84, 2018. Disponível em: http://www.sbcm.org.br/ojs3/index.php/rsbcm/article/view/336/304. Acesso em: 15 abr. 2020.

BRASIL. Ministério da Saúde. Resolução no 41, de 31 de outubro de 2018. Dispõe sobre as diretrizes para a organização dos cuidados paliativos, a luz dos cuidados continuados integrados, no âmbito do SUS. Diário Oficial da União, Brasília, DF, 31 out. 2018. Seção 1, p. 276.

CASSEL, J.B. et al. The business case for palliative care: translating research into program development in the U.S. Journal Pain Symptom Manage, v. 50, n. 6, p. 741-749, 2015. Disponível em: https://doi.org/10.1016/j.jpainsymman.2015.06.013. Acesso em: 15 abr. 2020.

CASTÔR, K. S. et al. Cuidados paliativos: perfil com olhar biopsicossocial dentre pacientes oncológicos. Brazilian Journal of Pain, v. 2, n. 1, p. 49-54, 2019. Disponível em: https://doi.org/10.5935/2595-0118.20190010. Acesso em: 15 abr. 2020.

CASTRA, M. L'émergence des soins palliatifs dans la médecine, une forme particulière de militantisme de fin de vie. Quaderni, v. 68, n. 1, p. 25-35, 2009. Disponível em: https://doi.org/10.4000/quaderni.260. Acesso em: 13 fev. 2020.

CORDEIRO, F.R.; KRUSE, M.H.L. Espaços de (final de) vida: estudo etnográfico em domicílios e estabelecimentos médico-sociais brasileiros e franceses. Revista Gaúcha de Enfermagem, v. 40, e20190065, 2019. Disponível em: https://doi.org/10.1590/1983-1447.2019.20190065. Acesso em: 10 jan. 2020.

FARINHOLT, P. et al. A Comparison of the Accuracy of Clinician Prediction of Survival Versus the Palliative Prognostic Index. Journal os pain and management symptom, v. 55, n. 3, p.792797, 2018. Disponível em: https://www.jpsmjournal.com/article/S0885-3924(17)306930/fulltext\# Acesso em: 29 nov. 2021.

FERNANDES, M. C. et al. Universidade e a extensão universitária: a visão dos moradores das comunidades circunvizinhas. Educação em Revista, v. 28, n. 4, p. 169-194, 2012. Disponível em: https://doi.org/10.1590/S0102-46982012000400007. Acesso em: 15 abr. 2020.

\section{FÓRUM DE PRÓ-REITORES DAS INSTITUIÇÕES PÚBLICAS DE EDUCAÇÃO SUPERIOR BRASILEIRAS. Política Nacional de Extensão Universitária. Manaus, 2012.}

GAMBLIN, V. et al. Les équipes mobiles de soins palliatifs: entre idéal normatif et réalité du terrain. Revue Internationale de Soins Palliatifs, v. 28, n. 1, p. 227-231, 2013. Disponível em: https://www.cairn.info/revue-infokara-2013-4-page-227.htm. Acesso em: 08 mar. 2020.

GARCIA, J. B. S.; RODRIGUES, R.F.; LIMA, S. F. A estruturação de um serviço de cuidados paliativos no Brasil: relato de experiência. Brazilian Journal of Anesthesiology, v. 64, n. 4, p. 286-291, 2014. Disponível em: https://doi.org/10.1016/j.bjan.2013.06.007. Acesso em: 08 mar. 2020.

GOIÁS. Assembleia Legislativa. Lei no 19.726 de 10, de julho de 2017. Institui a Política Estadual de Cuidados Paliativos. Diário Oficial do Estado, Goiânia, GO, 21 jul. 2017. 
GOMES, N. S. et al. Conhecimentos e práticas da enfermagem na administração de fluidos por via subcutânea. Revista Brasileira de Enfermagem, v. 70, n. 5, p. 1096-1105, 2017. Disponível em: https://doi.org.10.1590/0034-7167-2016-0424tt. Acesso em: 15 abr. 2020.

HOSPITAL ESCOLA DA UNIVERSIDADE FEDERAL DE PELOTAS. Carta de serviços ao cidadão. Pelotas: HE UFPEL/EBSERH, 2018.

INTERNATIONAL ASSOCIATION FOR HOSPICE AND PALLIATIVE CARE. Global Consensus based palliative care definition. Houston: The International Association for Hospice and Palliative Care, 2018. Disponível em: https://hospicecare.com/what-wedo/projects/consensus-based-definition-of-palliative-care/definition/.

KRUSE, M. H. L. et al. Cuidados paliativos: uma experiência. Revista HCPA, v. 27, n. 2, p. 49-52, 2007.

LAVAL, G. et al. Soins de support et réunions de concertation pluridisciplinaires (RCP): un nouveau défi? à propos de l'expérience d'un centre hospitalo-universitaire. Oncologie, v. 10, n. 1, p. 598-603, 2008. Disponível em: https://doi.org/10.1007/s10269-008-0923-2. Acesso em: 15 abr. 2020.

MACIEL, M.G.S. Avaliação do paciente em Cuidados Paliativos. In: CARVALHO, R.T; PARSONS, H.A. Manual de Cuidado Paliativos ANCP: ampliado e atualizado. 2 ed. São Paulo: ANCP, 2012.

MANFREDINI, L. L. Tradução e validação da Escala de Avaliação de Sintomas de Edmonton (ESAS) em pacientes com câncer avançado. 2014. 168 f. Dissertação (Mestrado em oncologia) - Fundação Pio XII, Hospital do Câncer de Barretos, Barretos, 2014.

MARCUCCI, F. C. I. et al. Implantação de uma Unidade de Cuidados Paliativos num hospital de média complexidade de Londrina - PR: relato de experiência. Revista de Saúde Pública do Paraná, v. 18, n. 1, p. 196-203, 2017. Disponível em: http://dx.doi.org/10.22421/15177130.2017v18n1p196. Acesso em: 08 mar. 2020.

MEDEIROS, J.B. Caracterização clínica e sociodemográfica de pacientes acompanhados pelo projeto de extensão "A consulta de enfermagem como instrumento de cuidado às pessoas com doenças que ameaçam a vida e suas famílias". $101 \mathrm{f}$. Trabalho de Conclusão de Curso (Graduação em Enfermagem), Universidade Federal de Pelotas, Pelotas, 2020.

MENEZES, R.A.; BARBOSA, P.C. A construção da "boa morte" em diferentes etapas da vida: reflexões em torno do ideário paliativista para adultos e crianças. Ciência \& Saúde Coletiva, v. 18, n. 9, p. 2653-2622, 2013. Disponível em: https://doi.org/10.1590/S141381232013000900020. Acesso em: 10 abr. 2020.

MICHELON, F. F. et al. Guia de integralização da extensão nos currículos dos cursos de graduação da Universidade Federal de Pelotas. Pelotas: Universidade Federal de Pelotas, 2019.

MORRISON. R.S. et al. Palliative care consultation teams cut hospital costs for medicaid beneficiaries. Health Affairs, v. 30, n. 3, p. 454-463, 2011. Disponível em: https://doi.org/10.1377/hlthaff.2010.0929. Acesso em: 12 jan. 2020. 
MURAD JÚNIOR, M. Avaliação de escore preditor de mortalidade em pacientes com tumor sólido avançado. 47 f. Dissertação de mestrado (Programa de Pós-Graduação em Ciências da Saúde do Adulto), Universidade Federal de Minas Gerais, Belo Horizonte, 2011.

OLIVEIRA, A. T. et al. Tempo entre a avaliação por uma equipe de consultoria em cuidados paliativos e o óbito de pacientes hospitalizados. In: Congresso de Cuidados Paliativos do Mercosul, 2019, Pelotas. Anais do IV Congresso de Cuidados Paliativos do Mercosul. Pelotas: Unidade Cuidativa da UFPEL, 2019. Disponível em: https://doity.com.br/anais/iv-congresso-cuidadospaliativos-mercosul-v3-2019/trabalho/95107

PAN, H. et al. Path modeling of knowledge, attitude and practice toward palliative care consultation service among Taiwanese nursing staff: a cross-sectional study. BMC Palliat Care, v. 42, n. 1, p. 1-9, 2017. Disponível em: https://doi.org/10.1186/s12904-017-0228-6. Acesso em: 2 abr. 2020

RIO GRANDE DO SUL. Assembleia Legislativa. Lei no 15.277, de 31 de janeiro de 2019. Institui a Política Estadual de Cuidados Paliativos e dá outras providências. Diário Oficial do Estado, Porto Alegre, RS, 31 jan. 2019.

SANTANA, M.T.E.A. et al. Adaptação transcultural e validação semântica de instrumento para identificação de necessidades paliativas em língua portuguesa. Einstein, v. 18, p. eAO5539, 2020. Disponível em: https://www.scielo.br/j/eins/a/jXYwndR7CyxFVSMMHBrPXMQ/?format=pdf\&lang=pt . Acesso em: 29 nov. 2021.

SILVA, G.; CECCHETTO, F. H. Dificuldades vivenciadas pelos profissionais de enfermagem na assistência ao paciente em cuidados paliativos. Revista de Enfermagem UFPI, v. 8, n. 3, p. 6469, 2019. Disponível em: https://doi.org/10.26694/2238-7234.8364-69. Acesso em: 14 abr. 2020.

STONER, K. L. et al. Intravenous versus subcutaneous drug administration: which do patients prefer? A Systematic Review. The Patient, v. 8, n. 1, p. 145-153, 2015. Disponível em: https://doi.org/10.1007/s40271-014-0075-y. Acesso em: 15 abr. 2020.

XAVIER, E.C.L et al. Diagnósticos de enfermagem em cuidados paliativos oncológicos segundo diagrama de abordagem multidimensional. Enfermagem em foco, v.10, n. 3, p. 7, p. 152-157, 2019. Disponível em: http://revista.cofen.gov.br/index.php/enfermagem/article/download/2109/569. Acesso em: 30 jul. 2020.

YAMADA, T. et al. A prospective, multicenter cohort study to validate a simple performance status-based survival prediction system for oncologists. Cancer, v. 123, n. 8, p. 1442-1452, 2017. Disponível em: https://acsjournals.onlinelibrary.wiley.com/doi/10.1002/cncr.30484. Acesso em: 30 jul. 2020.

Recebido em: 25/08/2020

Aceito em: 06/12/2021 